\title{
Normalization and centering of array-based heterologous genome hybridization based on divergent control probes
}

\author{
Brian J Darby ${ }^{1}$, Kenneth L Jones ${ }^{1,2}$, David Wheeler ${ }^{1}$ and Michael A Herman ${ }^{1 *}$
}

\begin{abstract}
Background: Hybridization of heterologous (non-specific) nucleic acids onto arrays designed for model-organisms has been proposed as a viable genomic resource for estimating sequence variation and gene expression in nonmodel organisms. However, conventional methods of normalization that assume equivalent distributions (such as quantile normalization) are inappropriate when applied to non-specific (heterologous) hybridization. We propose an algorithm for normalizing and centering intensity data from heterologous hybridization that makes no prior assumptions of distribution, reduces the false appearance of homology, and provides a way for researchers to confirm whether heterologous hybridization is suitable.

Results: Data are normalized by adjusting for Gibbs free energy binding, and centered by adjusting for the median of a common set of control probes assumed to be equivalently dissimilar for all species. This procedure was compared to existing approaches and found to be as successful as Loess normalization at detecting sequence variations (deletions) and even more successful than quantile normalization at reducing the accumulation of false positive probe matches between two related nematode species, Caenorhabditis elegans and C. briggsae. Despite the improvements, we still found that probe fluorescence intensity was too poorly correlated with sequence similarity to result in reliable detection of matching probe sequence.

Conclusions: Cross-species hybridizations can be a way to adapt genome-enabled tools for closely related nonmodel organisms, but data must be appropriately normalized and centered in a way that accommodates hybridization of nucleic acids with diverged sequence. For short, 25-mer probes, hybridization intensity alone may be insufficiently correlated with sequence similarity to allow reliable inference of homology at the probe level.
\end{abstract}

\section{Background}

Many organisms that are important components of most ecosystems are understudied at the genetic level because they lack useful genome-enabled resources. Hybridization of nucleic acids from non-model organisms onto DNA microarrays designed for closely related modelorganisms has been used as a potential alternative to building genomic resources for each species of interest. A variety of platforms and objectives in contemporary applications of heterologous ("cross-species") hybridizations, but the recurring challenge for each platform is to measure the effect of sequence dissimilarity on

\footnotetext{
* Correspondence: mherman@ksu.edu

'Ecological Genomics Institute, Division of Biology, Kansas State University, Manhattan, KS 66506, USA

Full list of author information is available at the end of the article
}

hybridization between the probes being used and the nucleic acids of the species being hybridized. For example, Gilad et al. [1] tested hybridization efficiency of microarrays spotted with amplicons from four primate species (including human) and showed that increasing sequence divergence resulted in reduced hybridization efficiency. Similarly, an array of expressed sequence tags (ESTs) from African cichlid fish (Astatotilapia burtoni) was used to test the validity of gene expression analysis on a variety of related teleost fish [2]. The number of spots (probe features) that were able to demonstrate differential gene expression decreased with increasing phylogenetic distance. The microarrays were subsequently used to assess gene expression from swordtail (Xiphophorus nigrensis) [3], which was estimated to be at the far edge of what was considered phylogenetically close
C Biomed Central

다 2011 Darby et al; licensee BioMed Central Ltd. This is an Open Access article distributed under the terms of the Creative Commons Attribution License (http://creativecommons.org/licenses/by/2.0), which permits unrestricted use, distribution, and reproduction in any medium, provided the original work is properly cited. 
enough to be reliable for cross-species hybridization on the cichlid arrays. Similar arrays developed from zebrafish (Danio) ESTs have been used with coral reef fish (Pomacentrus) cDNA [4]. In situ synthesized oligonucleotide arrays are an alternative to spotted cDNA microarrays and commonly used when the species of interest is closely related to a model organism for which a commercially designed chip is already available. For expression studies, it is common to screen probes for sequence conservation by first hybridizing heterologous gDNA, and secondly assessing gene expression by hybridizing experimental cDNA and analyzing only the accepted probes [5]. This strategy has been applied to examine gene expression of various genera of Brassicaceae on an array containing Arabidopsis thaliana probes, [5-7], expression of banana genes on a rice array [8], expression of horse genes on an array containing human probes [9], and expression of goat genes using a bovine array [10].

The preparation of heterologous hybridization data for analysis is problematic because probe binding is a result of multiple factors, including binding free-energy, selffolding, dimerization, and, importantly, sequence similarity or divergence [11]. Traditional approaches to analyzing heterologous hybridization data largely follow the techniques of array-based comparative genome hybridization (aCGH ) [12-14], which is the hybridization of gDNA to con-specific arrays for the detection of chromosomal or copy-number variations. These techniques can include local regression normalization and quantile normalization. However, the conventional normalization procedures designed for aCGH have the potential to result in the false appearance of homology if the probe signals from cross-species hybridizations violates the underlying assumptions of uniform statistical distributions due to sequence divergence. Several methods have been proposed to 'screen' probes and reduce the potential for false positives [15]: 1) accept only probes of a certain hybridization fluorescence threshold or overall intensity [5,16], 2) match probes from a reference genome to that of the target genome and only analyze probes of a certain sequence similarity [17], or 3) normalize the entire dataset using a suite of known conserved genes $[18,19]$. However, the significant challenge with normalizing intensity data based on conserved genes is that genes evolve at different rates for different lineages. Many non-model organisms have such little genomic sequence data known that identifying sets of genes with conserved sequences amongst a group of species is unreliable, if not impossible. We propose a normalization and centering approach that relies on universally diverged (non-conserved) probes and does not make any prior assumptions about the distribution of probe signal intensities.
Our initial objective in this study was to identify probes with conserved sequences that could be used as potential primer sites to allow amplification and subsequent cloning of orthologous genes of interest. Traditional normalization techniques led to numerous false positive matches, so we developed an algorithm for normalizing and centering intensity data of heterologous hybridization of gDNA for when the sequence divergence of species being used is unknown and a set of conserved genes is unreliable of impractical. Our approach uses built-in "control" probes from a plant (Arabidopsis thaliana) and a bacterium (Bacillus subtilis) that are available on the commercial Caenorhabditis elegans tiling array from Affymetrix ${ }^{\circledR}$ and assumed to be equivalently diverged from all nematode species used here (from the family Rhabditidae and Cephalobidae). This procedure is most applicable to oligonucleotide microarrays with universally diverged "control" features (e.g. from a different phylum or, preferably, domain than the target species) that have been hybridized with single dye-labeled gDNA of a related, but heterologous, species. The approach is tested for sensitivity and specificity using two isolates of C. elegans, one isolate of $C$. briggsae (for which the full genome sequence is available), and five other nematode species with little to no known sequence data beyond small subunit ribosomal RNA gene sequences (Figure 1). These nematodes are reasonable candidates for the study of genomic responses to environmental perturbation due to their ease of study in laboratory conditions and their phylogenetic proximity to the model species C. elegans. Furthermore, the species we selected all co-occur on the Konza Long-Term Ecological Research station [20], so it would be likely that researchers might wish to attempt heterologous array hybridization with a similar suite of nematodes. They represent the bacterial-feeding nematodes from the tallgrass prairie biome [21] and perform key ecosystem services such as regulating bacterial turnover and nutrient cycling in soil environments [22,23]. Renn et al. [2] suggested that heterologous hybridization is most successful when the species are diverged by less than 10 MYA, but also estimated that the approach could be used on species separated by as much as 25 MYA. Dating of the molecular divergence between these nematode species is inexact due to the lack of a robust and informative fossil record, but estimates of divergence time between $C$. elegans and C. briggsae has been narrowed down to 80-110 MYA following full genome sequencing [24]. Therefore, the two species from the family Cephalobidae (Acrobeloides sp. and Chiloplacus sp.) are so far diverged from $C$. elegans that they can serve as a negative control, while species within the family Rhabditidae (Oscheius tipulae, Oscheius sp. FVV-2, and Mesorhabditis sp.) serve as the species of interest to demonstrate the viability of this approach. 


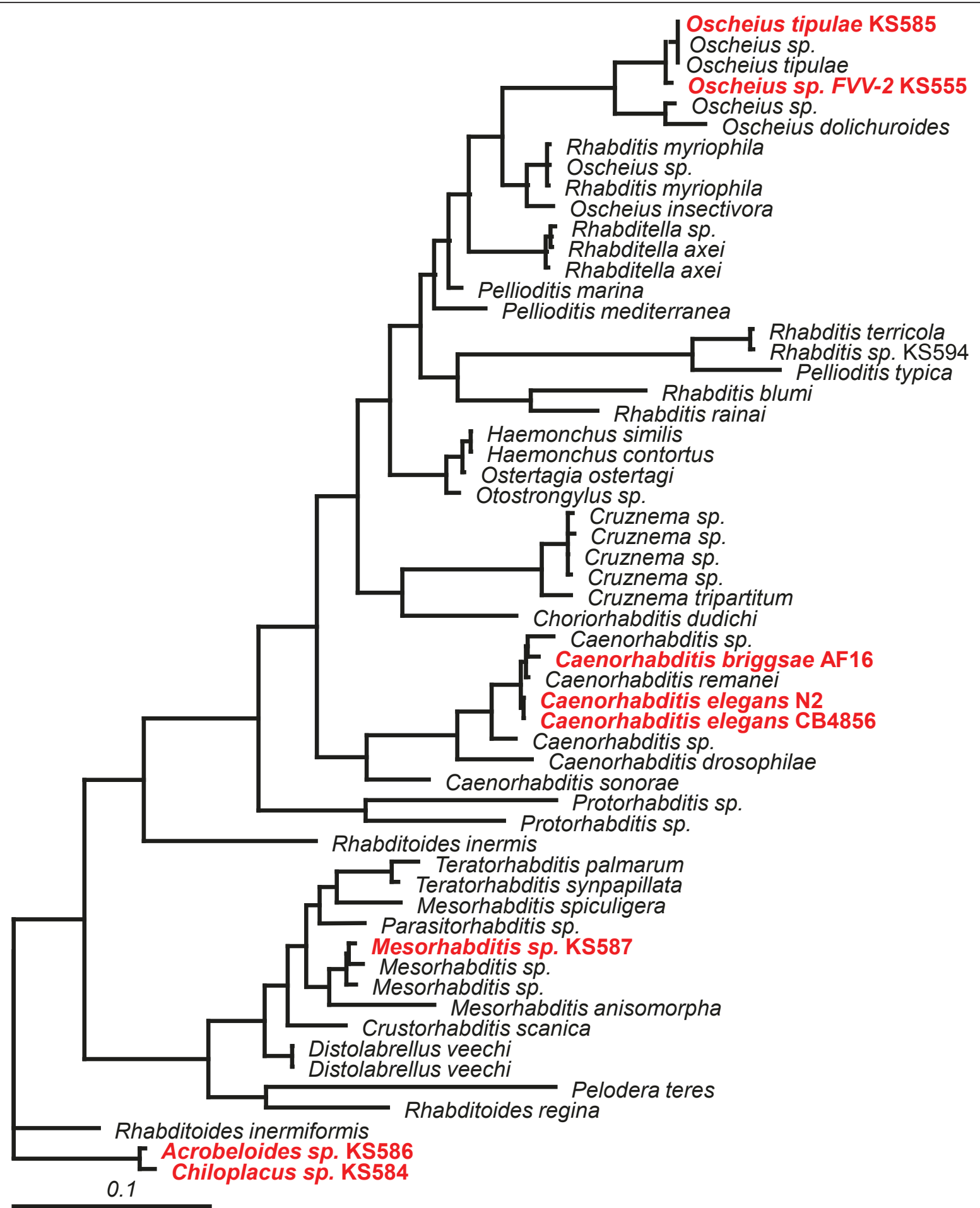

Figure 1 Phylogeny of study species. 18s ribosome RNA (small subunit) phylogeny of nematode species included in this study (in bold, red font), in comparison to 49 other species from the family Rhabditidae. Acrobeloides and Chiloplacus (from the family Cephalobidae) are used as outgroups. 


\section{Methods}

\section{gDNA and Hybridization conditions}

Strains used for hybridization included Caenorhabditis elegans (N2, C. elegans (CB4856), C. briggsae (AF16), and five species isolated from Konza Prairie, Riley County, Kansas (US): Oscheius tipulae (KS585) [Genbank:HQ130502], Oscheius sp. FVV-2 (KS555) [Genbank:HQ130503], Mesorhabditis sp. (KS587) [Genbank: HQ130505], Acrobeloides sp. (KS586) [Genbank: HQ130506], Chiloplacus sp. (KS584) [Genbank: HQ130507]. Genomic DNA was isolated from each species by phenol-chloroform extraction, labelled and hybridized onto the GeneChip ${ }^{\circledR}$ C. elegans Tiling 1.0R Array according to manufacturer's specification using two chips per species representing biological replicates. Arrays were imaged on GeneChip ${ }^{\circledR}$ Scanner 3000-7G and data extracted with GeneChip ${ }^{\mathbb{B}}$ Operating Software (GCOS) and analyzed using Tilling Analysis Software (TAS). Raw and processed data has been submitted to NCBI Gene Expression Omnibus [GEO:GSE23667].

\section{Analysis}

Raw background-subtracted probe intensities were accessed from Affymetrix ${ }^{\circledR}$ Tiling Analysis Software (TAS). Subsequent analyses were performed in Statistical Analysis Software, Release 9.1.3 (SAS Institute, Cary, NC, USA). Our approach utilizes "control" probes that are standard features of the Affymetrix C. elegans tiling chip that correspond to sequences in the Arabidopsis thaliana and Bacillus subtilis genomes. These features are used in gene expression studies to calibrate intensity with transcript concentration and to estimate $5^{\prime}$ and $3^{\prime}$ end bias, but are otherwise unused in genome hybridization. Probe-specific normalization was performed by first quantifying the relationship between $\log _{e}$-transformed probe intensity $\left(\ln \left(i_{c, s}\right)\right)$ of control probes $c$ from species $s$ and thermodynamic binding affinity $\Delta G_{37}$ (Gibb's free energy estimate, according to the nearest neighbour thermodynamic model of SantaLucia [25]):

$$
\ln \left(i_{c, s}\right)=\beta_{c, s} * \Delta \mathrm{G}_{37}+\alpha+e
$$

where $\alpha$ is the intercept and $e$ the error term (assumed to be normally distributed). We assume that gDNA from all nematode species was equally dissimilar from these control probes, therefore subtle differences in the relationship between binding efficiency of control probes to thermodynamic binding affinity most likely represents chip to chip variation. The resulting model parameters from (1) were used to normalize probe $p$ from each species $s$ to its final adjusted intensity (AI):

$$
\mathrm{AI}=\ln \left(i_{p, s}\right)-\left(\beta_{c, s} * \Delta \mathrm{G}_{37}\right)-\operatorname{median}(c, s)
$$

where the median intensity (median $(c, s)$ ) of all control probes $c$ from species $s$ was used as a phase shift to center all control probes around zero.

To characterize the relationship between probe intensity and the percent similarity, we make use of a dataset of candidate genes with potential ecologically relevant roles in nematode survival [26]. We selected 49 of the candidate genes of interest that had only one putative ortholog and confirmed that this suite of genes came from all six chromosomes (I: 3, II: 3, III, 6, IV: 8, V: 18, $\mathrm{X}: 11$ ) with a group GC content (min: $40.1 \%$, mean: 47.4\%, max: 65.8\%) that was representative of all probes in exon regions $(43 \% \pm 9.2 \mathrm{SD})$. We then aligned each probe from the C. elegans chip to its respective position in the C. briggsae homolog and computed the number of identical nucleotides.

\section{Results and Discussion}

\section{Conventional data transformation}

We initially analyzed the hybridization data using a conventional aCGH approach that included quantile normalization [12,13], scaling on a per-array basis to a common mean (500 intensity units), and a waveletbased smoothing of 50-bp bandwidth to 'de-noise' the data and accentuate regions of dissimilarity, or copynumber deviations, from baseline [14]. At first inspection, this conventional aCGH analysis resulted in probes of high signal intensity (comparable to that of C. elegans N2) for even non-Caenorhabditis species, especially around exonic regions (Figure 2D). However, attempts to amplify orthologous genes using PCR primers based on the exonic probes of high signal intensity (relative to C. elegans) were unsuccessful. It is possible that the greater probe intensity of exonic regions is not due to sequence similarity but to binding affinity, because probes in exon regions had a higher average GC-content $(43 \% \pm 9.2 \mathrm{SD})$ relative to probes in intron or intragenic regions $(34 \% \pm 10.4 \mathrm{SD})$. Thus, we hypothesized that quantile normalization of cross-species hybridization has the potential to result in the appearance of reliable data, but may in fact be a misleading representation of false positive probe matches due to artifacts during the data transformation process. Furthermore, smoothing can artificially inflate the intensity value of low-intensity probes that are adjacent to high intensity probes. Finally, the mean or median of heterologous hybridizations is naturally expected to be lower than homologous hybridizations because fewer sequences are perfect matches. Scaling all arrays to a common mean (or median) inflates nonspecific hybridization intensity relative to specific hybridization intensity. 


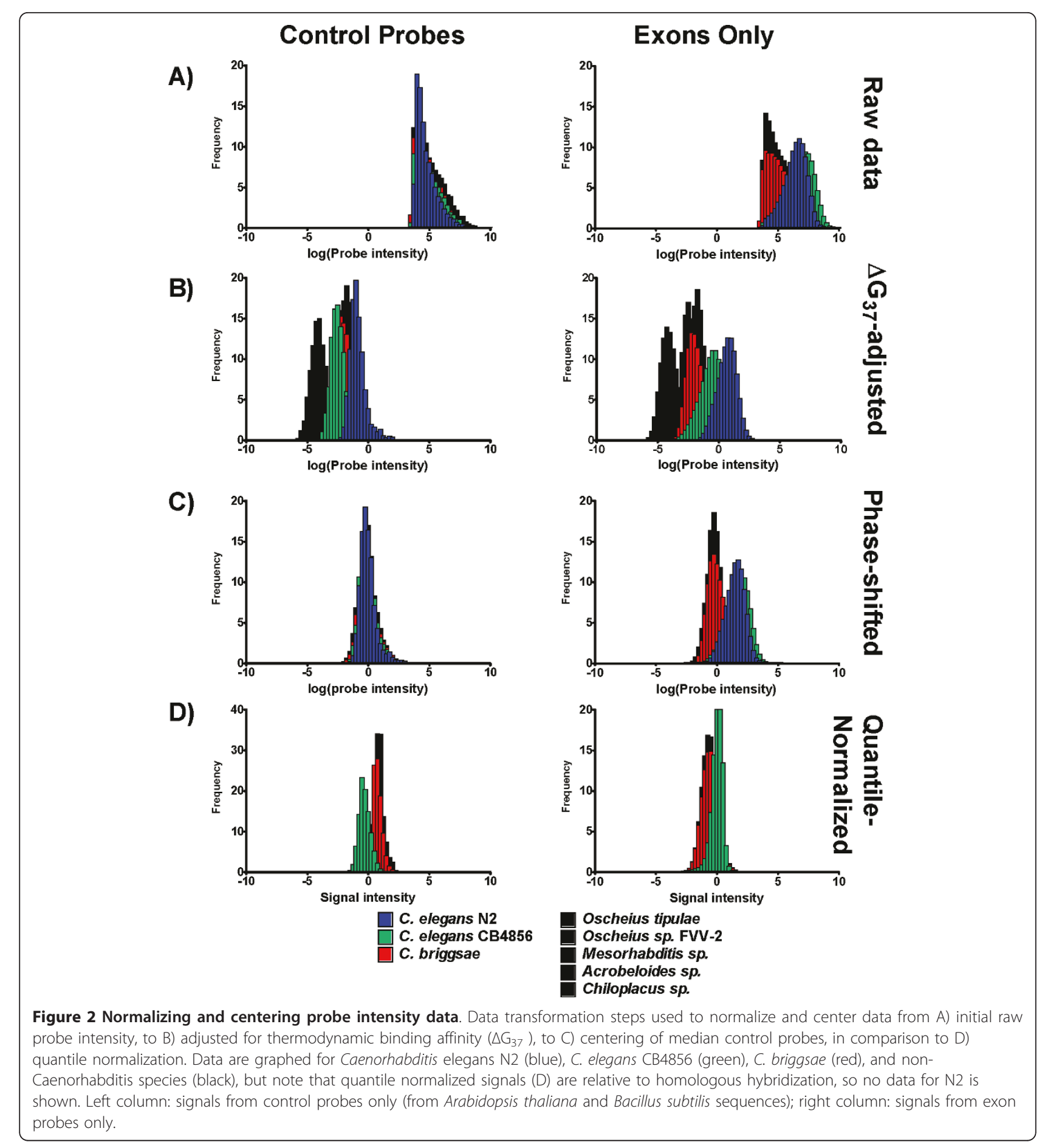

\section{Alternate normalization and centering}

To address the need for analyzing heterologous hybridization data with species for which the true genome-wide sequence similarity was unknown, we developed an alternative, probe-level normalization and centering. If normalization results in normally (or near normally) distributed probe intensities, the only remaining variability is likely to be predominantly random experimental error. The purpose of centering is to ensure that equivalent baseline hybridization is centered on a single common value. The raw probe intensity values (and statistical distribution) of $\log _{e}$-transformed control were nearly, but not exactly, similar between all chips (Figure 2A). After adjustment for the relationship between 
probe intensity and thermodynamic binding affinity (Figure 3), both target and control probes were uncentered, but normally distributed (Figure 2B). Finally, all probes were phase shifted to bring the median control probe intensity to zero (Figure $2 \mathrm{C}$ ). The result was that target probes from two Caenorhabditis elegans isolates averaged greater than zero (indicating specific binding signal), but the target probes from non-Caenorhabditis
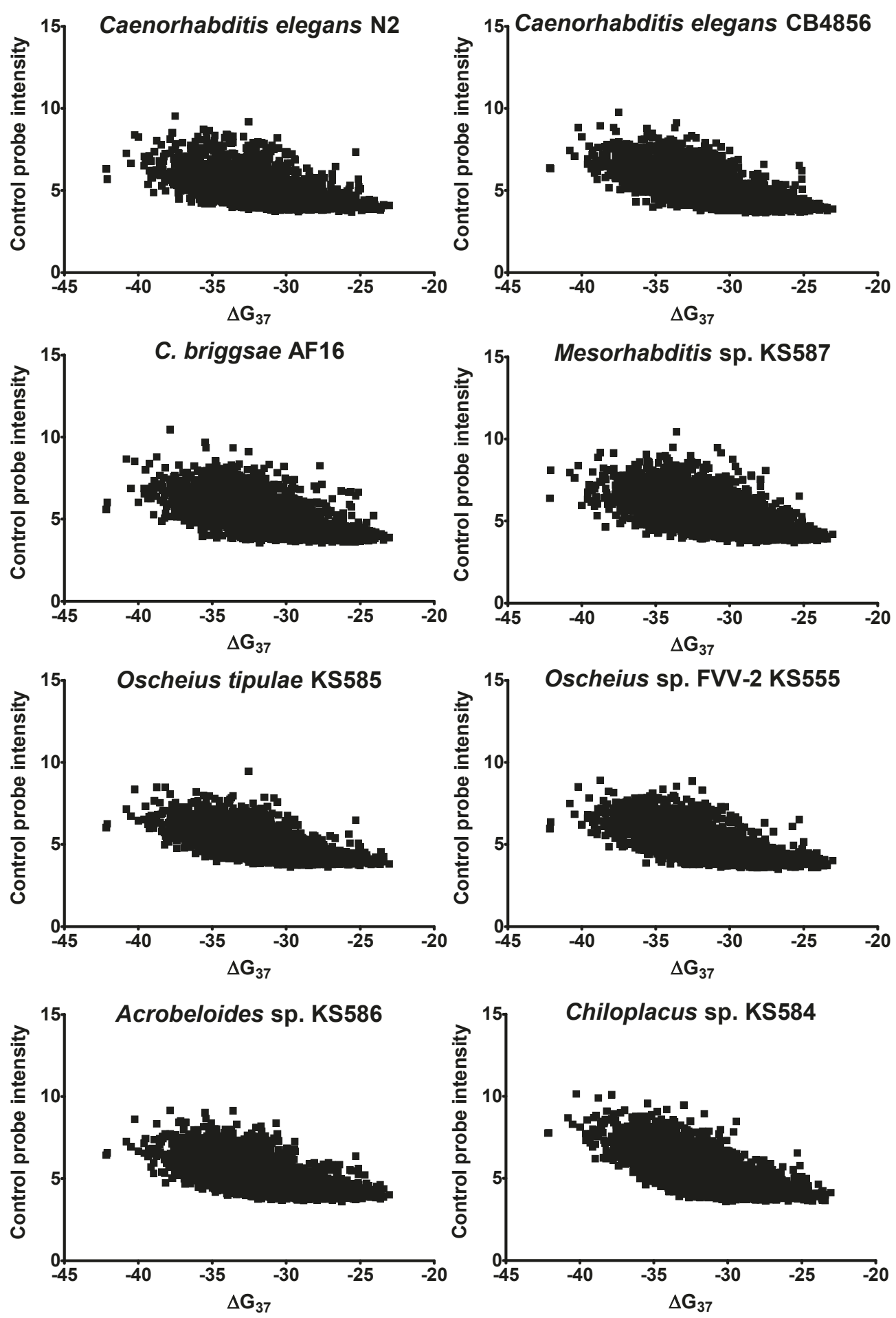

Figure 3 Thermodynamics of non-specific probe binding. The relationship between control probe intensity and thermodynamic binding affinity $\left(\Delta G_{37}\right)$ for each species tested that was used in the probe-level normalizing procedure. A) Caenorhabditis elegans N2, B) C. elegans (B4856, C) C. briggsae, D) Mesorhabditis sp., E) Oscheius tipulae, F) Oscheius sp. FW-2, G) Acrobeloides sp., H) Chiloplacus sp. 
species were centered near zero (indicating lack of specific binding). However, not all genes evolve at the same rates and it is possible that some genes within the genomes of the non-Caenorhabditis species are conserved and bind specifically to the C. elegans probes. If the average (normalized and centered) signal of all exon probes for a particular gene is significantly greater than zero, such a gene could reasonably be considered to be "conserved". As can be expected, the number of genes whose full set of exon probes average greater than zero decreases with increasing phylogenetic distance from $C$. elegans N2 (C. elegans CB4856: 17,399, C. briggsae AF16: 410, Oscheius tipulae KS585: 66, Oschieus sp. FVV-2 KS555: 61, Mesorhabditis sp. KS587: 41, Acrobeloides sp. KS586: 35, Chiloplacus sp. KS584: 30; t-test with Bonferroni correction for multiple tests). This was a first indication that sequence conservation in nonCaenorhabditis species may be too limited to permit detection of conserved regions.

\section{Test of sensitivity with con-specific hybridization}

To confirm that our normalization and centering approach was as capable of detecting conspecific variations as traditional normalization approaches, we were able to visually confirm 121 out of the 131 previously published deletions in C. elegans CB4856 [27]. To illustrate a representative case, Figure 4 shows a decrease in the ratio of probe values surrounding niDf94 $(\mathrm{V})$, a deletion of 3-kb of sequences in CB4856 (partially deleting

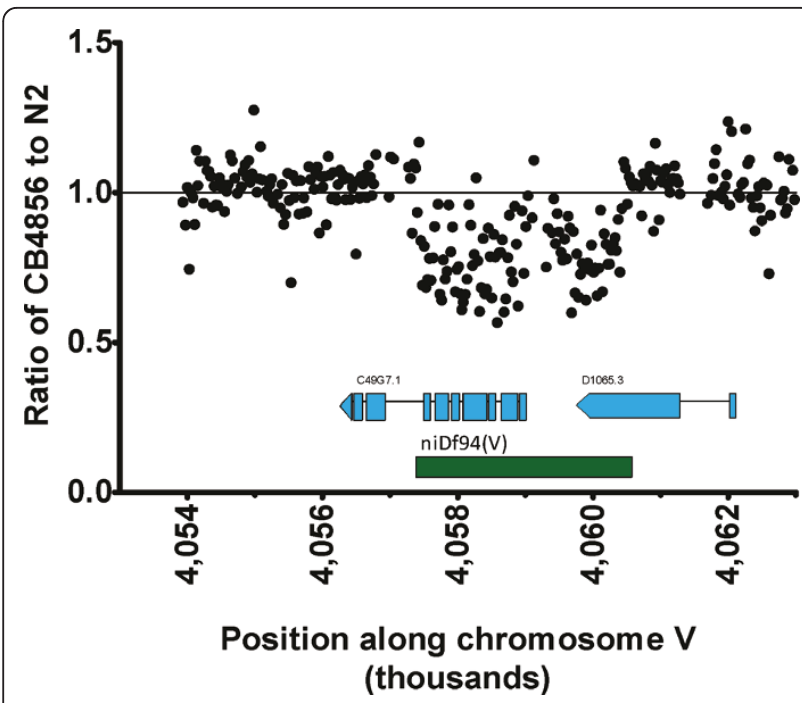

Figure 4 Confirmation of hybridization sensitivity. The ratio of C. elegans CB4856 to C. elegans N2 probe intensity values (following probe-level normalization and centering) in relation to the niDf94(V) deletion variant (of chromosome $\mathrm{V}$ ), which overlaps two

uncharacterized genes in the N2 genome, C49G7.1 and D1065.3a. The dip in probe intensity ratios below 1.0 is representative for the other 141 deletion variants previously identified [21]. the C49G7.1 and D1065.3 genes) that are present in N2. The few deletions we did not observe are likely due to small differences in genome coverage or different probe lengths ( 25 vs. 50 ) between the two platforms used.

\section{Test of specificity with cross-species hybridization}

Our initial objective of designing primers from suitable probes would involve identifying probes of conserved sequence as indicated by a fluorescence signal of significant intensity relative to that of known homologous binding from C. elegans. In order to do this we computed the ratio of each probe's signal intensity relative to that of C. elegans for each heterologous test species. We assumed that any 'threshold' ratio used to define a putatively homologous probe match could result in false positive matches, so we took advantage of the fully sequenced $C$. briggsae genome to define a signal intensity ratio that would minimize the rate of incorrectly identified sequence matches. We expected two major sources of variability in the intensity of C. briggsae gDNA hybridized to C. elegans, 1) multiple occurrences of the same probe sequence within either the C. elegans or C. briggsae genome, and 2) sequence divergence between the two species. First, we computed the number of times that each 25-mer probe sequence occurred in the C. elegans and C. briggsae genomes (Figure 5A). As expected, probe intensity from C. elegans gDNA was positively related to frequency, while probe intensity of C. briggsae gDNA was generally unaffected (Figure 5B). Although many probes occur multiple times in the $C$. briggsae genome, it is a small portion of the over three million probes on the array and does not have a large effect on the overall results. Next, we determined the nucleotide similarity at each corresponding probe position between C. elegans and C. briggsae for 49 single copy genes, identified in a previous study as being potentially important for survival in different bacterial environments. Quantile normalization resulted in poor discrimination of true positive probe matches (Figure $6 \mathrm{~A}$ ) and a relative high false positive rate at all potential signal intensity ratios (Figure 6B). Our alternate normalization and centering improved the discrimination of true perfect match probes (Figure $6 \mathrm{C}$ ), and lowered the ratio of false-matches to perfect-matches at some threshold ratios (Figure 6D). Although the values resulting from quantile normalization and from our proposed normalization and centering are comparable, they are not necessarily on equivalent scales. For example, selecting a signal intensity ratio from quantile normalization of 0.6 detects 14 true positive matches, but results in over 155 false positive mismatches. A ratio of 1.1 from our proposed alternative normalization and centering based on universally diverged control probes also detects 14 true positive matches, but results in only 34 


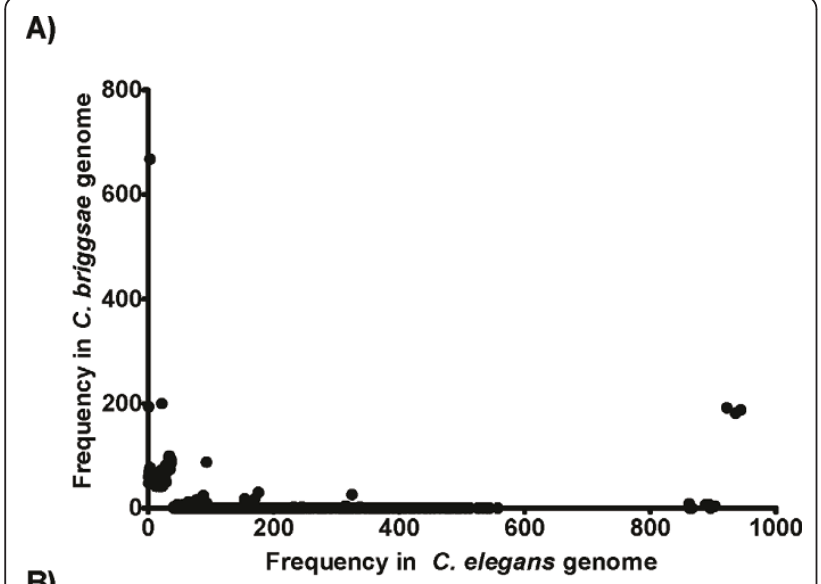

B)

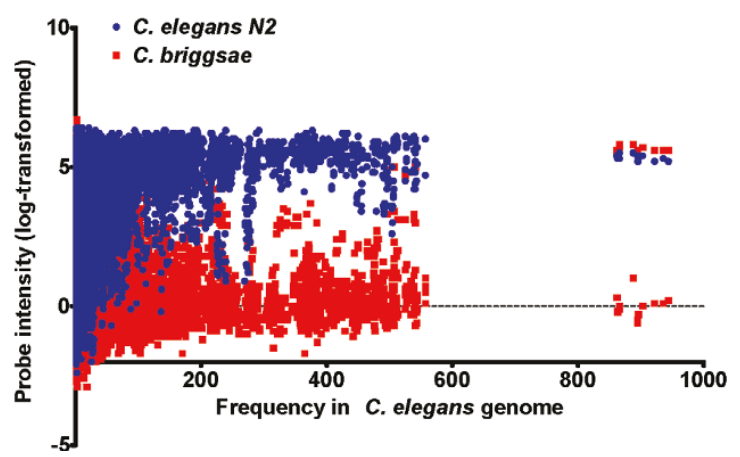

Figure 5 Effect of multiple-copy probes on binding intensity. A) Comparison of probe frequency found in C. elegans and C. briggsae genomes. B) Probe intensity of $C$. elegans (blue) and C. briggsae (red) gDNA related to probe frequency in C. elegans genome.

mismatches. Despite an over 4.5-fold reduction, the ultimate false positive detection rate is still high.

\section{Conclusions}

Cross-species hybridization has been proposed as a way to adapt genome-enabled tools developed for model organisms to closely related non-model relatives. However, we (present work) and others [18,19] have shown that the data must be appropriately normalized and centered to control for sequence divergence. Ultimately, we found that probe intensity alone was a poor predictor of sequence similarity and can result in false inferences of homology. Our findings largely support the recent results of Machado and Renn [18] who also found that the ability to detect genes decreased below 90\% sequence identity between three species of Drosophila. The major difference in our approach is that Machado and Renn normalize based on the 100 or 1000 most conserved genes (assumed to be equivalently similar for all species of interest), while we propose normalizing and centering based on control, non-target probes (assumed to be equivalently dissimilar for all species tested). Both approaches appear to be valid for their respective purposes, but our approach might be more applicable in the absence of enough genomic sequence data to identify an a priori set of conserved genes. The lack of universally dissimilar probes on the spotted chip of Machado and Renn [18] prevent us from applying our technique on their data, and the lack of genomic sequence data amongst our species prevent us from applying their technique on our data. However, we can nonetheless predict that the microarrays printed with PCR products $\sim 500$ bp long [18] are likely to be more sensitive and specific to their targets than the 25-mer probes used in the Affymetrix platform presented here. Single mismatches may have a more adverse affect on the binding of short, 25-bp probes, than long, $\sim 500 \mathrm{bp}$ probes. Hybridization of gDNA onto microarrays is currently the standard technique to validate probes on gene chips for expression analysis in cross-species applications. One commonly used procedure [5] hybridizes heterologous gDNA from a non-model organism onto an existing 25-mer GeneChip ${ }^{\circledR}$ designed for a model organism and masks all probe sets except those with at least one probe feature whose hybridization intensity is above a predefined threshold intensity. Our analysis suggests that, either with or without probe-level normalization and centering, a large number of non-specific control probes can still have a relatively high hybridization intensity compared to specific probes (Figure 2A). Furthermore, even if the threshold intensity were set relative to target genome hybridization, we show that a significant fraction of probe features at all threshold intensities could likely be false-positives. Thus, we fear that a cross-species hybridization algorithm to mask chips for gene expression may still permit a large number of false positive probe sets into the analysis. It is for this reason that studies utilizing cross-species hybridization for microarray gene expression profiles must be especially diligent with replication and validation. For example, Pavlidis et al. [28] found that a minimum of five biological replicates generated stable gene expression profiles. Unfortunately, recent studies using crossspecies hybridization on microarrays with short probes either include no replication or insufficiently validate their microarray results with qPCR $[8,9]$. We suggest that cross-species microarray hybridizations introduce a degree of uncertainty beyond what is typical for conspecific hybridizations, and thus require more robust quality control measures than would be normally adopted for con-specific hybridization.

Genomic DNA controls are essential to ensure the most reliable interpretation of heterologous hybridization applications, such as gene expression profiles. Our strategy for normalization and centering of cross-species array data is meant to be used to identify reliable probe 
A)

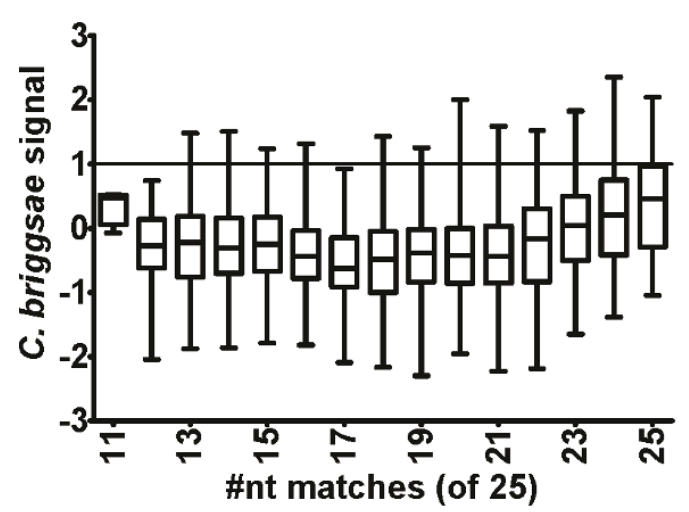

C)

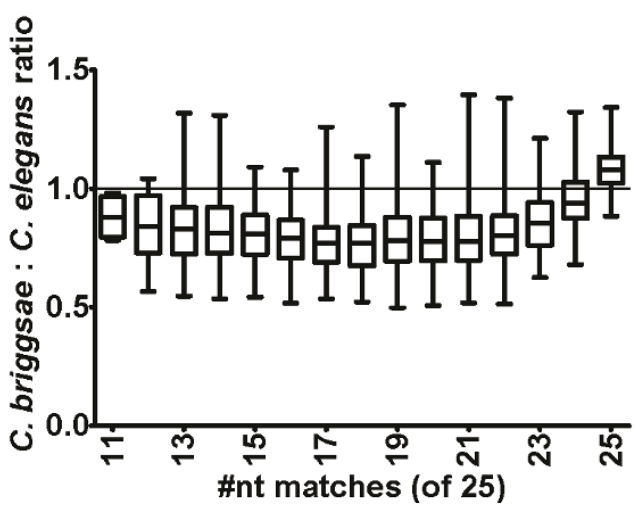

B)

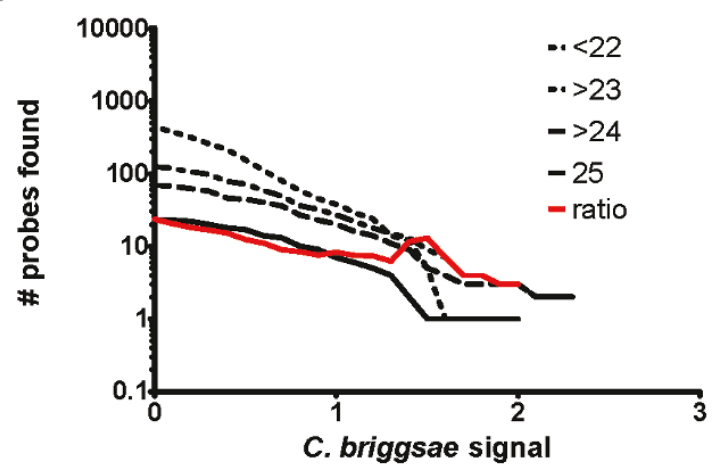

D)

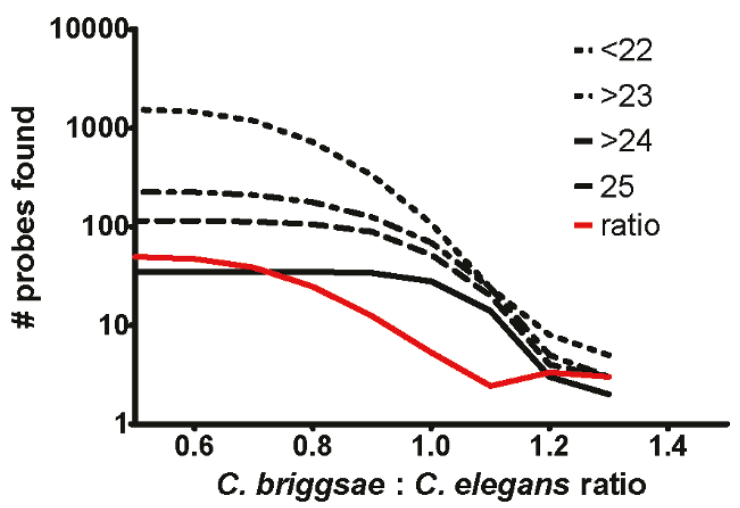

Figure 6 Heterologous probe binding of Caenorhabditis briggsae. A) Quantile-normalized C. briggsae probe intensity (relative to C. elegans N2) as a function of probe similarity (nucleotide matches out of 25 from all probes of the 49 candidate genes with one putative ortholog). B) Accumulation of false $(<22)$, true $(25)$, and semi-true $(>23,>24)$ positive probe matches at threshold ratios below 1.3 from previous panel (Figure $6 \mathrm{~A})$. Note that no threshold ratio is available to distinguish a greater number of semi-true positive $(>23 \mathrm{nt})$ than false $(<22)$ matches. C) and D) As in A) and B), but for alternative normalized and centered probe signal intensities proposed here. Red lines in $B$ and $D$ represent the ratio of mismatch probes (<25 nt) to perfect match probes (= $25 \mathrm{nt})$.

intensity values that could be utilized in downstream applications, such as finding regions of sequence similarity or for gene expression analysis. Our method is not necessarily meant to be used as a normalization procedure per se, although we could imagine that such an approach could be developed based on the analyses presented here. One such approach would be first to build universal control probe sets into the microarray of interest using random oligonucleotides or sequences derived from universally diverged taxa such as prokaryotes for eukaryotic arrays or vice-versa. Secondly, hybridize both homologous genomic DNA (from the species used to design the array) and heterologous (from the species of interest) genomic DNA onto the arrays being used (either dual-labelled mixtures onto the same chip or single-labelled pools onto separate chips) to compare probe intensity using the "control" based normalization and centering approach presented here. Finally, test the mean signal of a gene's exon probes against "zero" (with an appropriate correction for multiple comparisons). Only those genes whose complement of exon probes are statistically greater than zero can be considered "conserved" enough for use. Based upon our analyses, the number of these "conserved" genes decreases rapidly with phylogenetic distance and suggests that for distantly related taxa nonarray based approaches might be more appropriate and cost effective.

\section{Acknowledgements}

We thank Mandar Deshpande for assistance with hybridization protocol and imaging and the Caenorhabditis Genetics Center for providing the Caenorhabditis strains. This work was supported by NSF grant EF-0723862 to $\mathrm{MAH}$. Thanks also to Ted Morgan, two anonymous reviewers, and the members of the Ecological Genomics Institute at Kansas State University for discussions and helpful comments on the manuscript, and the Gene Expression Facility at Kansas State University for use of the facility. 


\section{Author details}

${ }^{1}$ Ecological Genomics Institute, Division of Biology, Kansas State University, Manhattan, KS 66506, USA. ²Department of Biochemistry and Molecular Genetics, University of Colorado School of Medicine, Aurora, CO 80045, USA.

\section{Authors' contributions}

$\mathrm{KL}$ designed the project and carried out the hybridizations, BJD performed the analysis and drafted the manuscript, DW directed the analysis and assisted in interpretation of data, MAH participated in project design, and coordination of the project, and helped draft the manuscript. All authors read and approved the final manuscript.

\section{Received: 16 September 2010 Accepted: 21 May 2011}

Published: 21 May 2011

\section{References}

1. Gilad Y, Rifkin SA, Bertone P, Gerstein M, White KP: Multi-species microarrays reveal the effect of sequence divergence on gene expression profiles. Genome Research 2005, 15(5):674-680.

2. Renn S, Aubin-Horth N, Hofmann H: Biologically meaningful expression profiling across species using heterologous hybridization to a CDNA microarray. BMC Genomics 2004, 5(1):42.

3. Cummings ME, Larkins-Ford J, Reilly CRL, Wong RY, Ramsey M, Hofmann HA: Sexual and social stimuli elicit rapid and contrasting genomic responses. Proceedings of the Royal Society B: Biological Sciences 2008, 275(1633):393-402

4. Kassahn KS, Caley MJ, Ward AC, Connolly AR, Stone G, Crozier RH: Heterologous microarray experiments used to identify the early gene response to heat stress in a coral reef fish. Molecular Ecology 2007, 16(8):1749-1763.

5. Hammond J, Broadley M, Craigon D, Higgins J, Emmerson Z, Townsend H, White $\mathrm{P}$, May S: Using genomic DNA-based probe-selection to improve the sensitivity of high-density oligonucleotide arrays when applied to heterologous species. Plant Methods 2005, 1(1):10.

6. Hammond JP, Bowen HC, White PJ, Mills V, Pyke KA, Baker AJM, Whiting SN, May ST, Broadley MR: A comparison of the Thlaspi caerulescens and Thlaspi arvense shoot transcriptomes. New Phytologist 2006, 170(2):239-260

7. Morinaga SI, Nagano AJ, Miyazaki S, Kubo M, Demura T, Fukuda H, Sakai S, Hasebe M: Ecogenomics of cleistogamous and chasmogamous flowering: genome-wide gene expression patterns from cross-species microarray analysis in Cardamine kokaiensis (Brassicaceae). Journal of Ecology 2008, 96(5):1086-1097

8. Davey M, Graham N, Vanholme B, Swennen R, May S, Keulemans J: Heterologous oligonucleotide microarrays for transcriptomics in a nonmodel species; a proof-of-concept study of drought stress in Musa. BMC Genomics 2009, 10(1):436

9. Graham NS, Clutterbuck AL, James N, Lea RG, Mobasheri A, Broadley MR, May ST: Equine transcriptome quantification using human GeneChip arrays can be improved using genomic DNA hybridisation and probe selection. The Veterinary Journal 2010, 186(3):323-327.

10. Faucon F, Rebours E, Bevilacqua C, Helbling JC, Aubert J, Makhzami S, Dhorne-Pollet S, Robin S, Martin P: Terminal differentiation of goat mammary tissue during pregnancy requires the expression of genes involved in immune functions. Physiol Genomics 2009, 40(1):61-82.

11. Pozhitkov A, Noble PA, Domazet-Loso T, Nolte AW, Sonnenberg R, Staehler P, Beier M, Tautz D: Tests of rRNA hybridization to microarrays suggest that hybridization characteristics of oligonucleotide probes for species discrimination cannot be predicted. Nucleic Acids Research 2006, 34(9).

12. Bolstad BM, Irizarry RA, Astrand M, Speed TP: A comparison of normalization methods for high density oligonucleotide array data based on variance and bias. Bioinformatics 2003, 19(2):185-193.

13. Irizarry RA, Bolstad BM, Collin F, Cope LM, Hobbs B, Speed TP: Summaries of Affymetrix GeneChip probe level data. Nucl Acids Res 2003, 31(4):e15.

14. Hsu L, Self SG, Grove D, Randolph T, Wang K, Delrow JJ, Loo L, Porter P: Denoising array-based comparative genomic hybridization data using wavelets. Biostat 2005, 6(2):211-226

15. Bar-Or C, Czosnek H, Koltai H: Cross-species microarray hybridizations: a developing tool for studying species diversity. Trends in Genetics 2007, 23(4):200-207.
16. Degletagne C, Keime C, Rey B, de Dinechin M, Forcheron F, Chuchana P, Jouventin P, Gautier C, Duchamp C: Transcriptome analysis in non-model species: a new method for the analysis of heterologous hybridization on microarrays. BMC Genomics 2010, 11(1):344.

17. Bar-Or C, Bar-Eyal M, Gal T, Kapulnik Y, Czosnek H, Koltai H: Derivation of species-specific hybridization-like knowledge out of cross-species hybridization results. BMC Genomics 2006, 7(1):110.

18. Machado H, Renn S: A critical assessment of cross-species detection of gene duplicates using comparative genomic hybridization. BMC Genomics 2010, 11(1):304

19. Renn S, Machado H, Jones A, Soneji K, Kulathinal R, Hofmann H: Using comparative genomic hybridization to survey genomic sequence divergence across species: a proof-of-concept from Drosophila. BMC Genomics 2010, 11(1):271.

20. Jones KL, Todd TC, Wall-Beam JL, Coolon JD, Blair JM, Herman MA: Molecular Approach for Assessing Responses of Microbial-Feeding Nematodes to Burning and Chronic Nitrogen Enrichment in a Native Grassland. Molecular Ecology 2006, 15(9):2601-2609.

21. Todd TC, Powers TO, Mullin PG: Sentinel nematodes of land-use change and restoration in tallgrass prairie. Journal of Nematology 2006, 38(1):20-27.

22. Freckman DW: Bacterivorous Nematodes and Organic-Matter Decomposition. Agriculture Ecosystems \& Environment 1988, 24(1-3):195-217.

23. Ferris $H$, Bongers $T$ : Nematode Indicators of Organic Enrichment. Journal of Nematology 2006, 38(1):3-12

24. Stein LD, Bao Z, Blasiar D, Blumenthal T, Brent MR, Chen N, Chinwalla A, Clarke L, Clee C, Coghlan A, et al: The Genome Sequence of Caenorhabditis briggsae: A Platform for Comparative Genomics. PLoS Biol 2003, 1(2):e45.

25. SantaLucia J: A unified view of polymer, dumbbell, and oligonucleotide DNA nearest-neighbor thermodynamics. Proceedings of the National Academy of Sciences of the United States of America 1998, 95(4):1460-1465.

26. Coolon JD, Jones KL, Todd TC, Carr BC, Herman MA: Caenorhabditis elegans Genomic Response to Soil Bacteria Predicts EnvironmentSpecific Genetic Effects on Life History Traits. PLoS Genetics 2009, 5(6): e1000503.

27. Maydan JS, Flibotte S, Edgley ML, Lau J, Selzer RR, Richmond TA, Pofahl NJ, Thomas JH, Moerman DG: Efficient high-resolution deletion discovery in Caenorhabditis elegans by array Comparative Genomic Hybridization. Genome Research 2007, 17(3):337-347.

28. Pavlidis P, Li Q, Noble WS: The effect of replication on gene expression microarray experiments. Bioinformatics 2003, 19(13):1620-1627.

doi:10.1186/1471-2105-12-183

Cite this article as: Darby et al:: Normalization and centering of arraybased heterologous genome hybridization based on divergent control probes. BMC Bioinformatics 2011 12:183.

\section{Submit your next manuscript to BioMed Central and take full advantage of:}

- Convenient online submission

- Thorough peer review

- No space constraints or color figure charges

- Immediate publication on acceptance

- Inclusion in PubMed, CAS, Scopus and Google Scholar

- Research which is freely available for redistribution

Submit your manuscript at www.biomedcentral.com/submit
C Biomed Central 